\title{
2-Aminopteridin-4-one derivatives from 2, 3-dicyanopyrazine
}

\author{
Masaru Tada,* Daiju Shiono, and Shinsuke Tanaka \\ Department of Chemistry, Waseda University, Shinjuku, Tokyo 169-8555, Japan \\ E-mail:mtada@waseda.jp
}

(received 15 Jul 05; accepted 26 Aug 05; published on the web 31 Aug 05)

\begin{abstract}
2-Allylamino-3-cyanopyrazine (3) was transformed into 1-allylpteridin-4-one-2-thiones (4) by the reaction with isothiocyanates, and the thiones 4 were thermally converted into 2-allylthiopteridin-4-ones (5) by 3,3-sigmatropic rearrangement. The allylthio group of 5 was replaced by a butylamino group to give 2-butylaminopteridin-4-ones (6).
\end{abstract}

Keywords: Pteridine synthesis, nucleophilic substitution of pyrazine, S-Cope rearrangement

\section{Introduction}

2-Aminopteridin-4-one (1) constitutes a core structure in the commonly occurring coenzymes such as biopterin, ${ }^{1}$ folic acid, ${ }^{1,2}$ and methanopterin. ${ }^{3}$ Previously, we have investigated reactions of 2,3-dicyanopyrazine, ${ }^{4}$ which was conveniently prepared from diaminomaleonitrile and glyoxal, and its derivatization to pteridine derivatives. ${ }^{5} 2,3$-Dicyanopyrazine (2) is easily converted into 2-allylamino-3-cyanopyrazine $(3)^{6}$ by the simple treatment with allylamine. In this paper we would like to report the synthesis of 2-aminopteridin-4-one derivatives, starting from 2,3-dicyanopyrazine (2).

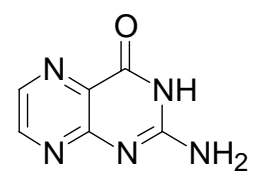

1

\section{Results and Discussion}

2-Allylamino-3-cyanopyrazine (3) was conveniently prepared by the treatment of 2,3-dicyanopyrazine (2) with allylamine ${ }^{6}$ and then the cyanopyrazine $\mathbf{3}$ was converted to 1-allyl-3-phenyl- (4a), 1-allyl-3-butyl- (4b), or 1-allyl-3-methyllpteridin-4-one-2-thione (4c) in 
$69-88 \%$ yields by the reactions with the corresponding isothiocyanates followed by hydrolysis of the intermediate imines.

Pteridinethione derivatives $\mathbf{4 a}, \mathbf{4 b}$ and $\mathbf{4 c}$ were heated to $180-198^{\circ} \mathrm{C}$ to cause the 3,3-sigmatropic rearrangement, and the thermal equilibration produced allylthio derivatives $\mathbf{5 a}, \mathbf{5 b}$ and $5 \mathrm{c}$ as components of the equilibrium mixtures (40\%, 23\%, and $21 \%$ respectively) along with the intact starting materials. Thermal treatment of the isolated products $\mathbf{5 a}, \mathbf{5 b}$ and $\mathbf{5 c}$ gave the same equilibrium mixtures of 4's and 5's by the re-equilibration, and therefore this transformation is reversible. Unfortunately, the equilibria between $\mathrm{N}$-allyl derivatives 4's and S-allyl derivatives 5's favor the former, but the starting materials 4's can be efficiently recovered and subjected to another thermal isomerization. The treatment of compounds 5 with butylamine substitutes the allylthio group with butylamine to produce 2-butylamino-3-phenyl- (6a), 2-butylamino-3-butyl(6b), and 2-butylamino-3-methylpteridin-4-ones (6c) in 60-85\% yields. Other alkylamines react in the same manner though only butylamine is shown here as an example (Scheme 1).
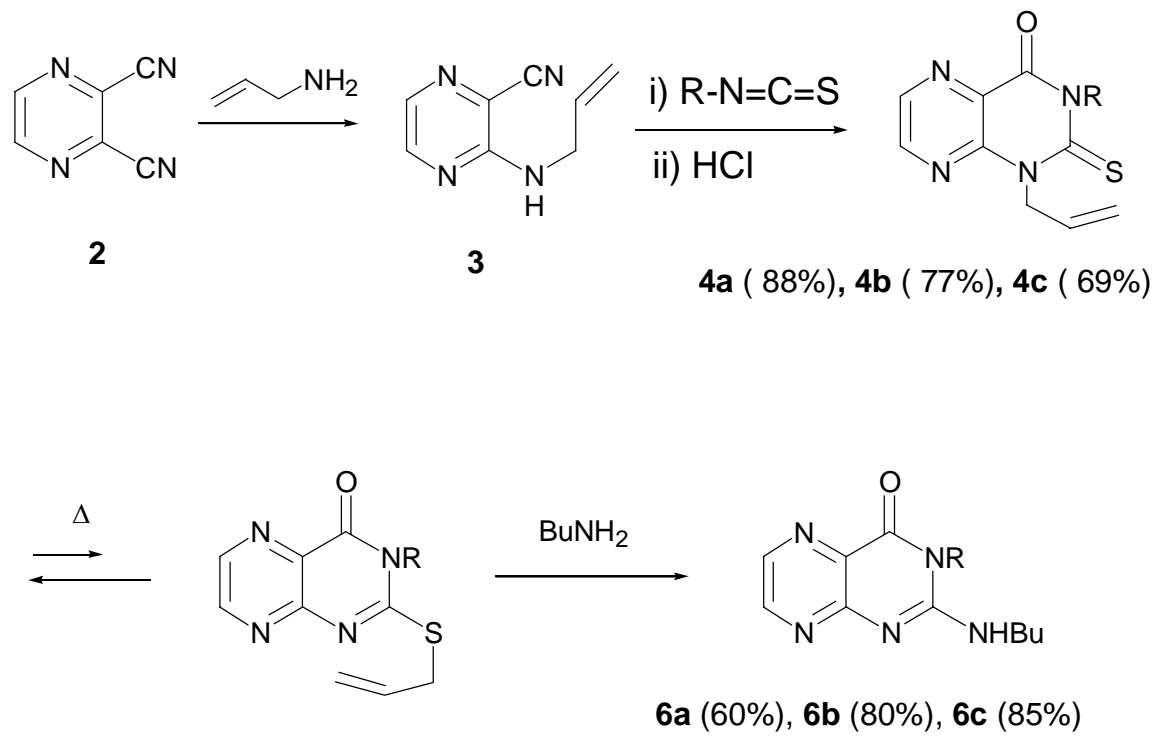

$5 a, 5 b, 5 c$

$4 a-6 a(R=P h) ; 4 b-6 b(R=B u) ; 4 c-6 c(R=M e)$

Scheme 1. Derivation of aminopteridin-4-ones by S-Cope rearrangement.

Structures of thiones $\mathbf{4 a - 4 c}$ and the allylthio derivatives $\mathbf{5 a - 5 c}$ were assigned straightforwardly by the NMR signals due to the protons on the pyrazine ring and by the appropriate shift of the signals of the allylic protons due to the rearrangement, $\mathrm{N}-\mathrm{CH}_{2}$ 5.58-5.59 for 4's and $\mathrm{S}-\mathrm{CH}_{2}$ 3.96-4.08 for 5's. The substitution $\mathbf{5} \rightarrow \mathbf{6}$ is evident from the replacement of the signals due to the allylthio group by those due to the butylamino group.

The removal of the allyl group from thiones 4 was attempted by the previously reported methods such as palladium catalysis, ${ }^{7}$ hydrogen abstraction by thiyl radical, ${ }^{8,9}$ and allyl group transfer to methyl chloroformate. ${ }^{10}$ All these attempts to remove the allyl group at this stage were 
unsuccessful probably due to the steric hindrance for the approach of a reagent. The present thio-Cope rearrangement is a promising method for the removal of $\mathrm{N}$-allyl group and the introduction of amino group by the nucleophilic substitution is efficient. The procedure reported in this paper is a convenient and simple route to the synthesis of 2-aminopteridin-4-one derivatives, although the appropriate structural modifications are required for the favorable S-Cope rearrangement of thiones 4 .

\section{Experimental Section}

Synthesis of 1-allylpteridin-4-one-2-thiones (4a, 4b and 4c). In a nitrogen-flushed flask were placed sodium hydride (60\% dispersion with mineral oil, $176 \mathrm{mg}, 4.4 \mathrm{mmol})$ and $640 \mathrm{mg}$ (4.0 mmol) of 2-allylamino-3-cyanopyrazine (3) ${ }^{6}$ dissolved in $20 \mathrm{ml}$ of THF, and the mixture was stirred for $10 \mathrm{~min}$ at room temperature. Phenylisothiocyanate $(810 \mathrm{mg}, 6.0 \mathrm{mmol})$ was added dropwise to the mixture and the reaction mixture was stirred for $2.5 \mathrm{~h}$ at room temperature. After the addition of $2 \mathrm{M} \mathrm{HCl}$, washing with chloroform, and neutralization with aq. $\mathrm{NaHCO}_{3}$, the product was extracted three times with chloroform. Conventional work up of the extract gave crude product of 1-allyl-3-phenylpteridin-4-imino-2-one. This imino derivative was hydrolyzed to 4a by refluxing for $1.0 \mathrm{~h}$ in $2 \mathrm{M} \mathrm{HCl}$. The reaction mixture was extracted three times with chloroform after neutralization with saturated aq. $\mathrm{NaHCO}_{3}$. The crude product was recrystallized from ethanol to give $1.04 \mathrm{~g}$ of 1-allyl-3-phenylpteridin-4-one-2-thione (4a) (88\%). The thiones $\mathbf{4 b}$ and $4 \mathrm{c}$ were prepared by the same procedure in 77 and $69 \%$ yields.

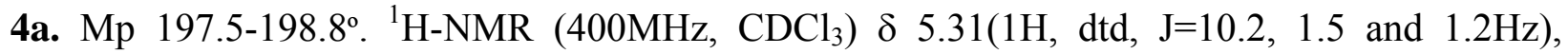
5.38(1H, dtd, $\mathrm{J}=17.5,1.2$ and $1.2 \mathrm{~Hz}), 5.59(2 \mathrm{H}, \mathrm{ddd}, \mathrm{J}=5.6,1.2$ and $1.2 \mathrm{~Hz}), 6.03(1 \mathrm{H}$, ddt, $\mathrm{J}=17.5$, 10.0 and $5.6 \mathrm{~Hz}), 7.24(2 \mathrm{H}, \mathrm{m}), 7.50(1 \mathrm{H}, \mathrm{m}), 7.56(2 \mathrm{H}, \mathrm{m}), 8.63(1 \mathrm{H}, \mathrm{d}, \mathrm{J}=2.2 \mathrm{~Hz}), 8.76(1 \mathrm{H}, \mathrm{d}$, $\mathrm{J}=2.2 \mathrm{~Hz}) ;{ }^{13} \mathrm{C}-\mathrm{NMR}\left(150 \mathrm{MHz}, \mathrm{CDCl}_{3}\right)$ 50.8, 119.4, 128.1, 128.9, 129.5, 129.7, 130.0, 130.3, 141.5, 148.1, 148.4, 157.9, 178.0; IR $\left(\mathrm{CHCl}_{3}\right)$ 3012, 3007, 1710, 1579, 1547, 1475, 1428, $1385 \mathrm{~cm}^{-1}$; MS(FAB) $\mathrm{m} / \mathrm{z}=297\left(\mathrm{M}^{+}+\mathrm{H}\right)$. Anal. C, 60.44; H, 3.75; N, 18.82. Calcd for $\mathrm{C}_{15} \mathrm{H}_{12} \mathrm{~N}_{4} \mathrm{OS}$ : C, 60.79; H, 4.08; N, 18.91 .

4b. Mp 144.0-145.1 ${ }^{\circ} .{ }^{1} \mathrm{H}-\mathrm{NMR}\left(400 \mathrm{MHz}, \mathrm{CDCl}_{3}\right) \delta 0.99(3 \mathrm{H}, \mathrm{t}, \mathrm{J}=7.3 \mathrm{~Hz}), 1.45(2 \mathrm{H}, \mathrm{tq}, \mathrm{J}=7.6$ and $7.3 \mathrm{~Hz}), 1.79(2 \mathrm{H}, \mathrm{tt}, \mathrm{J}=7.8$ and $7.6 \mathrm{~Hz}), 4.65(2 \mathrm{H}, \mathrm{t}, \mathrm{J}=7.8 \mathrm{~Hz}), 5.27(1 \mathrm{H}, \mathrm{d}, \mathrm{J}=10.5 \mathrm{~Hz}), 5.29(1 \mathrm{H}, \mathrm{d}$, $\mathrm{J}=17.1 \mathrm{~Hz}), 5.58(2 \mathrm{H}, \mathrm{d}, \mathrm{J}=5.4 \mathrm{~Hz}), 6.01(1 \mathrm{H}, \mathrm{ddd}, \mathrm{J}=17.1,10.5$ and $5.4 \mathrm{~Hz}), 8.65(1 \mathrm{H}, \mathrm{d}, \mathrm{J}=2.2 \mathrm{~Hz})$, 8.70(1H, d, J=2.2Hz); ${ }^{13} \mathrm{C}-\mathrm{NMR}\left(150 \mathrm{MHz}, \mathrm{CDCl}_{3}\right)$ 13.7, 20.1, 28.2, 48.7, 50.5, 118.9, 129.2, 130.5, 141.5, 147.8, 147.9, 157.7, 176.8; IR $\left(\mathrm{CHCl}_{3}\right)$ 3014, 1700, 1549, 1482, 1438, $1420 \mathrm{~cm}^{-1}$; $\mathrm{MS}(\mathrm{FAB}) \mathrm{m} / \mathrm{z}=277\left(\mathrm{M}^{+}+\mathrm{H}\right)$. Anal. C, 56.47; H, 5.66; N, 20.20. Calcd for $\mathrm{C}_{13} \mathrm{H}_{16} \mathrm{~N}_{4} \mathrm{OS}$ : C, 56.50; H, 5.84; N, 20.27.

4c. Mp 172.0-174.2 $.{ }^{1} \mathrm{H}-\mathrm{NMR}\left(400 \mathrm{MHz}, \mathrm{CDCl}_{3}\right) \delta 3.94(3 \mathrm{H}, \mathrm{s}), 5.27(1 \mathrm{H}, \mathrm{dtd}, \mathrm{J}=9.9,1.5$ and $1.0 \mathrm{~Hz}), 5.30(1 \mathrm{H}$, dtd, $\mathrm{J}=16.5,1.5$ and $1.0 \mathrm{~Hz}), 5.59(2 \mathrm{H}$, ddd, $\mathrm{J}=5.6,1.5$, and $1.5 \mathrm{~Hz}), 6.01(1 \mathrm{H}$, ddt, $\mathrm{J}=16.5,9.9$ and $5.6 \mathrm{~Hz}), 8.67(1 \mathrm{H}, \mathrm{d}, \mathrm{J}=2.1), 8,73(1 \mathrm{H}, \mathrm{d}, \mathrm{J}=2.1 \mathrm{~Hz}) ;{ }^{13} \mathrm{C}-\mathrm{NMR}\left(150 \mathrm{MHz}, \mathrm{CDCl}_{3}\right)$ $36,3,50.6,119.1,129.0,130.4,141.6,147.8,148.0,158.2$, 177.2; IR $\left(\mathrm{CHCl}_{3}\right)$ 3015, 1705, 1550, 
1484, 1438, $1419 \mathrm{~cm}^{-1}$; HRMS(FAB) m/z=235.0670. Calcd for $\left(\mathrm{C}_{10} \mathrm{H}_{10} \mathrm{~N}_{4} \mathrm{OS}+\mathrm{H}\right) \mathrm{m} / \mathrm{z}=235.0653$. Anal. C, 51.21; H, 4.01; N, 23.62. Calcd for $\mathrm{C}_{10} \mathrm{H}_{10} \mathrm{~N}_{4} \mathrm{OS}$ : C, 51.27; H, 4.30; N, 23.91.

Thermal isomerization of 1-allylpteridin-4-one-2-thiones (4a, 4b and 4c) to 3-allylthiopteridin-4-ones (5a, 5b and 5c). Crystalline thione 4a (69 $\mathrm{mg}, 0.2 \mathrm{mmol}$ ) was placed in a glass tube and heated to the melting point $\left(198^{\circ}\right)$ for $2 \mathrm{~h}$ under argon atmosphere. Chromatography of the residue on silica gel eluted with hexane-ethyl acetate (2:1) yielded allylthio derivative 5a in $40 \%$ yield and mostly recovered $\mathbf{4 a}$. Thione $\mathbf{4 b}$ was heated at $180^{\circ}$ in refluxing o-dichlorobenzene for 48 $\mathrm{h}$, and thione $\mathbf{4 c}$ was heated at $190^{\circ}$ for $20 \mathrm{~h}$ in o-dichlorobenzene using a sealed glass tube. Allylthio derivatives $\mathbf{5 b}$ and $\mathbf{5 c}$ were obtained from $\mathbf{4 b}$ and $\mathbf{4 c}$ in the same work up in $23 \%$ and $21 \%$ respectively.

5a. ${ }^{1} \mathrm{H}-\mathrm{NMR}\left(400 \mathrm{MHz}, \mathrm{CDCl}_{3}\right) \delta 3.96(2 \mathrm{H}$, ddd, $\mathrm{J}=5.1,1.2$ and $1.2 \mathrm{~Hz}), 5.18(1 \mathrm{H}, \mathrm{dtd}, \mathrm{J}=10.0,1.2$ and $1.5 \mathrm{~Hz}), 5.34(1 \mathrm{H}, \mathrm{dtd}, \mathrm{J}=17.0,1.2$ and $1.5 \mathrm{~Hz}), 5.93(1 \mathrm{H}$, ddt, $\mathrm{J}=17.0,10.0$ and $5.1 \mathrm{~Hz}), 7.34(2 \mathrm{H}$, $\mathrm{m}), 7.58(3 \mathrm{H}, \mathrm{m}), 8.76(1 \mathrm{H}, \mathrm{d}, \mathrm{J}=2.2), 8.90(1 \mathrm{H}, \mathrm{d}, \mathrm{J}=2.2) ;{ }^{13} \mathrm{C}-\mathrm{NMR}\left(150 \mathrm{MHz}, \mathrm{CDCl}_{3}\right) 36.5,119.9$, 128.6, 129.2, 130.5, 130.8, 131.4, 131.9, 141.9, 143.9, 150.5, 160.8, 164.0: IR $\left(\mathrm{CHCl}_{3}\right) 3015$, $1714,1559,1539,1526,1404 \mathrm{~cm}^{-1} ; \operatorname{HRMS}(\mathrm{FAB}) \mathrm{m} / \mathrm{z}=297.0841$. Calcd for $\left(\mathrm{C}_{15} \mathrm{H}_{12} \mathrm{~N}_{4} \mathrm{OS}+\mathrm{H}\right)$ $\mathrm{m} / \mathrm{z}=297.0812$.

5b. ${ }^{1} \mathrm{H}-\mathrm{NMR}\left(400 \mathrm{MHz}, \mathrm{CDCl}_{3}\right) \delta 1.00(3 \mathrm{H}, \mathrm{t}, \mathrm{J}=7.3), 1.47(2 \mathrm{H}, \mathrm{tq}, \mathrm{J}=7.6$ and 7.3$), 1.80(2 \mathrm{H}$, tt, $\mathrm{J}=8.1$ and 7.6$), 4.08(2 \mathrm{H}$, ddd, $\mathrm{J}=6.8,1.2$ and $1.2 \mathrm{~Hz}), 4.19(2 \mathrm{H}, \mathrm{t}, \mathrm{J}=8.1 \mathrm{~Hz}), 5.26(1 \mathrm{H}, \mathrm{dtd}, \mathrm{J}=10.0$, 1.0 and $1.0 \mathrm{~Hz}), 5.44(1 \mathrm{H}, \mathrm{dtd}, \mathrm{J}=17.1,1.2$ and $1.0 \mathrm{~Hz}), 6.03(1 \mathrm{H}$, ddt, $17.1,10.0$ and $6.8 \mathrm{~Hz})$, 8.72(1H, d, J=2.0Hz), 8.85(1H, d, J=2.0Hz); ${ }^{13} \mathrm{C}-\mathrm{NMR}\left(150 \mathrm{MHz}, \mathrm{CDCl}_{3}\right)$ 13.8, 20.3, 29.8, 35.6, 45.4, 120.0, 131.5, 131.6, 143.6, 150.1, 153.2, 160.5, 162.3; IR $\left(\mathrm{CHCl}_{3}\right)$ 3019, 3015, 1700, 1550, $1532,1411 \mathrm{~cm}^{-1}$; HRMS(FAB) m/z=277.1118. Calcd for $\left(\mathrm{C}_{13} \mathrm{H}_{16} \mathrm{~N}_{4} \mathrm{OS}+\mathrm{H}\right) \mathrm{m} / \mathrm{z}=277.1123$.

5c. ${ }^{1} \mathrm{H}-\mathrm{NMR}\left(400 \mathrm{MHz}, \mathrm{CDCl}_{3}\right) \delta 3.69(3 \mathrm{H}, \mathrm{s}), 4.09(2 \mathrm{H}, \mathrm{d}, \mathrm{J}=6.8), 5.26(1 \mathrm{H}, \mathrm{dd}, \mathrm{J}=9.8$ and $1.5 \mathrm{~Hz})$, $5.45(1 \mathrm{H}, \mathrm{dd}, \mathrm{J}=16.6$ and $1.5 \mathrm{~Hz}), 6.03(1 \mathrm{H}$, ddt, $\mathrm{J}=16.6,9.8$ and $6.8 \mathrm{~Hz}), 8.73(1 \mathrm{H}, \mathrm{d}, \mathrm{J}=2.4 \mathrm{~Hz})$, 8.86(1H, d, J=2.4Hz); ${ }^{13} \mathrm{C}-\mathrm{NMR}\left(150 \mathrm{MHz}, \mathrm{CDCl}_{3}\right)$ 31.5, 35.9, 120.3, 131.6, 131.8, 143.9, 144.0, 150.5, 161.1, 163.1; IR $\left(\mathrm{CHCl}_{3}\right) 3006,1700,1550,1534,1419 \mathrm{~cm}^{-1} ; \operatorname{HRMS}(\mathrm{FAB}) \mathrm{m} / \mathrm{z}=235.0672$. Calcd for $\left(\mathrm{C}_{10} \mathrm{H}_{10} \mathrm{~N}_{4} \mathrm{OS}+\mathrm{H}\right) \mathrm{m} / \mathrm{z}=235.0653$.

Synthesis of 2-butylaminopteridin-4-ones (6a, 6b and 6c). 2-Allylthio-3-phenylpyteridin-4-one (5a) $(20 \mathrm{mg}, 0.068 \mathrm{mmol})$ was placed in a nitrogen-flushed flask and treated with $0.14 \mathrm{ml}$ ( $1.4 \mathrm{mmol}$ ) of butylamine dissolved in $0.5 \mathrm{ml}$ of THF and the mixture was refluxed for $24 \mathrm{~h}$. Concentration and chromatography of the reaction mixture on silica gel eluted with ethyl acetate-ethanol (10:1) gave 2-butylamino-3-phenylpteridin-4-one (6a) in 60\% yield. The same procedure gave $\mathbf{6 b}$ and $\mathbf{6 c}$ from $\mathbf{5 b}$ and $\mathbf{5 c}$ in 80 and $85 \%$ yield, respectively.

6a. ${ }^{1} \mathrm{H}-\mathrm{NMR}\left(400 \mathrm{MHz}, \mathrm{CDCl}_{3}\right) \delta 0.91(3 \mathrm{H}, \mathrm{t}, \mathrm{J}=7.3 \mathrm{~Hz}), 1.38(2 \mathrm{H}, \mathrm{q}, \mathrm{J}=7.3 \mathrm{~Hz}), 1.59(2 \mathrm{H}$, tt, J=7.1 and $7.3 \mathrm{~Hz}), 3.47(2 \mathrm{H}, \mathrm{td}, \mathrm{J}=7.1$ and $5.4 \mathrm{~Hz}), 4.35(1 \mathrm{H}, \mathrm{bs}, \mathrm{NH}), 7.25-7.29(2 \mathrm{H}, \mathrm{m}), 7.52-7.61(3 \mathrm{H}, \mathrm{m})$, 8.43(1H, d, J=2.2Hz), 8.65(1H, d, J=2.2Hz); ${ }^{13} \mathrm{C}-\mathrm{NMR}\left(150 \mathrm{MHz}, \mathrm{CDCl}_{3}\right)$ 13.7, 20.0, 31,1, 42.0, 128.6, 129.9, 139.6, 131.0, 133.7, 140.3, 150.0, 151.9, 156.0, 161.1; IR $\left(\mathrm{CHCl}_{3}\right)$ 3434, 3000, 1701, $1564,1412 \mathrm{~cm}^{-1}$; HRMS(FAB) $\mathrm{m} / \mathrm{z}=296.1519$. Calcd for $\left(\mathrm{C}_{16} \mathrm{H}_{17} \mathrm{~N}_{5} \mathrm{O}+\mathrm{H}\right) \mathrm{m} / \mathrm{z}=296.1511$.

6b. ${ }^{1} \mathrm{H}-\mathrm{NMR}\left(400 \mathrm{MHz}, \mathrm{CDCl}_{3}\right) \delta 0.98(3 \mathrm{H}, \mathrm{t}, \mathrm{J}=7.3 \mathrm{~Hz}), 0.99(3 \mathrm{H}, \mathrm{t}, \mathrm{J}=7.3 \mathrm{~Hz}), 1.44(2 \mathrm{H}$, tq, J=7.1 and 7.3$), 1.46(2 \mathrm{H}, \mathrm{tq}, \mathrm{J}=7.1$ and $7.3 \mathrm{~Hz}), 1.70(2 \mathrm{H}, \mathrm{tt}, \mathrm{J}=7.3$ and $7.1 \mathrm{~Hz}), 1.73(2 \mathrm{H}, \mathrm{tt}, \mathrm{J}=7.6$ and 
7.1Hz), 3.68(2H, td, $J=7.3$ and $5.4 \mathrm{~Hz}), 4.10(2 \mathrm{H}, \mathrm{t}, \mathrm{J}=7.6 \mathrm{~Hz}), 5.10(1 \mathrm{H}, \mathrm{bs}, \mathrm{NH}), 8.47(1 \mathrm{H}, \mathrm{d}$, $\mathrm{J}=2.0 \mathrm{~Hz}), 8.69(1 \mathrm{H}, \mathrm{d}, \mathrm{J}=2.0 \mathrm{~Hz}) ;{ }^{13} \mathrm{C}-\mathrm{NMR}\left(150 \mathrm{MHz}, \mathrm{CDCl}_{3}\right)$ 13.7, 13.8, 20.1, 20.2, 29.4, 31.3, 41.5, 42.3, 129.1, 140.1, 149.9, 151.7, 155.4, 161.4; IR $\left(\mathrm{CHCl}_{3}\right)$ 3480, 3014, 1700, 1580, 1540, $1471 \mathrm{~cm}^{-1}$; HRMS(FAB) m/z=276.1801. Calcd for $\left(\mathrm{C}_{14} \mathrm{H}_{21} \mathrm{~N}_{5} \mathrm{O}+\mathrm{H}\right) \mathrm{m} / \mathrm{z}=276.1824$.

6c. ${ }^{1} \mathrm{H}-\mathrm{NMR}\left(400 \mathrm{MHz}, \mathrm{CDCl}_{3}\right) \delta 0.99(3 \mathrm{H}, \mathrm{t}, \mathrm{J}=7.3 \mathrm{~Hz}), 1.46(2 \mathrm{H}, \mathrm{tq}, \mathrm{J}=7.4$ and $7.3 \mathrm{~Hz}), 1.71(2 \mathrm{H}$, tt, $\mathrm{J}=7.3$ and $7.3 \mathrm{~Hz}), 3.58(3 \mathrm{H}, \mathrm{s}), 3.69(2 \mathrm{H}, \mathrm{td}, \mathrm{J}=7.3$ and $5.3 \mathrm{~Hz}), 3.86(1 \mathrm{H}, \mathrm{bs}, \mathrm{NH}), 8.49(1 \mathrm{H}, \mathrm{d}$, $\mathrm{J}=2.2 \mathrm{~Hz}), 8.71(1 \mathrm{H}, \mathrm{d}, \mathrm{J}=2.2 \mathrm{~Hz}) ;{ }^{13} \mathrm{C}-\mathrm{NMR}\left(150 \mathrm{MHz}, \mathrm{CDCl}_{3}\right)$ 13.7, 20.1, 28.1, 31.2, 42.4, 128.9 , 140.4, 150.0, 152.1, 155.3, 161.4; IR $\left(\mathrm{CHCl}_{3}\right)$ 3474, 3013, 3001, 1696, 1583, 1565, $1476 \mathrm{~cm}^{-1}$; $\operatorname{HRMS}(F A B) \mathrm{m} / \mathrm{z}=234.1372$. Calcd for $\left(\mathrm{C}_{11} \mathrm{H}_{15} \mathrm{~N}_{5} \mathrm{O}+\mathrm{H}\right) \mathrm{m} / \mathrm{z}=234.1355$.

\section{References}

1. Wood, H. C. S. Comprehensive Organic Chemistry, Haslam, E., Ed.; Pergamon: Oxford, 1979; Vol. 5, pp 508-515.

2. (a)Temple, Jr. C.; Montgomery, J. A. Chemistry and Biochemistry of Folates; Blackley, J. A.; Benkovic, S. J., Eds; John-Wiley and Sons, 1984; Vol. 1, pp 61-120.

3. DiMarco, A. A.; Bobik, T. A.; Wolfe, R. S. Ann. Rev. Biochem. 1990, 59, 355.

4. (a) Hirano, H.; Tada, M. J. Heterocycl. Chem. 1981, 18, 905. (b) Hirano, H.; Lee, R. Tada, M. J. Heterocycl. Chem. 1982, 19, 1409. (c) Tsuzuki, K; Tada, M. J. Heterocycl. Chem. 1985, 22 , 1365; 1986, 23, 1299.

5. (a) Tada, M.; Asawa, Y.; Igarashi, M. J. Heterocycl. Chem. 1997, 34, 9373. (b) Tada, M.; Shimamura, T.; Suzuki, T. Heterocycles 2003, 60, 2511.

6. Tada, M.; Wada, M. J. Chem. Research (S) 2003, 648.

7. Guibé, F. Tetrahedron 1998, 54, 2967.

8. Feelding, A. J.; Roberts, B. P. Tetrahedron Lett. 2001, 42, 4061.

9. Bertrand, M. P.; Escaubeut, S. Gastaldi, S.; Timokhin, V. I. Chem. Comunn. 2002, 216.

10. Janetka, J. W.; Furnes, M. S.; Zhong, X.Y.; Coop, A. ; Folk, J. E. ; Mattson, M. V.; Jacobson, A. E.; Rice, K. C. J. Org. Chem. 2003, 68, 3976. 